\title{
Review on Driverless Traffic from Management Perspective
}

\author{
Tingting Chen ${ }^{1}$, Fei Chen ${ }^{1}$ and Cheng Chen ${ }^{1}$ \\ ${ }^{1}$ School of Transportation, Southeast University, 210096 No.2 Sipailou, Nanjing, P.R. China
}

\begin{abstract}
The move towards automated driving is gaining ground. This paper reviews the development process of self-driving technology and discusses the safety and efficiency advantages of autonomous vehicles. The discussion shows that the existing traffic management system, including transport infrastructures and regulations, should be changed accordingly to maximize the advantages of autonomous driving. Thus, this paper subsequently gives an insight of the traffic management from three aspects: fully self-driving traffic infrastructures, mixed traffic infrastructures and regulations. First, it is summarized in detail what should be adjusted in intersections, parking lots, pedestrian crossings, ramps, signs and markings. With the transformation of traffic infrastructures, the advantages of driverless car will be more pronounced on account of increased capacity, reduced delay and land use. Also, this paper indicates that the implementations of strict product liability for self-driving car manufacturers and no-fault tort liability for users are applicable to automated vehicle accidents.
\end{abstract}

\section{Introduction}

\subsection{Development of automated driving}

Autonomous car is also referred as self-driving car or wheeled robot, which can sense surroundings via sensor without human input, and be driven by the intelligent autopilot based on computer system. The concept was firstly raised by American industrial designer Norman Bel Geddes in 1939. Then, the United States, Britain and other countries carried on the authentication. In 1977, the first autonomous car was made by S.Tsugawa and his colleagues in Mechanical Engineering Laboratory, Tsukuba, Japan. Of which the speed can get to $30 \mathrm{~km} / \mathrm{h}$.

With the deepening of research, the definition of automation is being consummated continuously. In 2013, NHTSA defined it into five levels: no automation, function-specific automation, combined function automation, limited self-driving automation, full selfdriving automation [1]. Now, the development of general self-driving car is in the third stage, while the "Autopilot" of Tesla is in the fourth stage and Google self-driving car the fifth. It is estimated that self-driving car will be partially used in 2020, and will fully come into society up to 2040 [2].

\subsection{Impacts}

Automated driving is usually thought as "disruptive technologies"[3]. It not only brings great convenience to traffic, but also influences existing laws 、 industrial structure、 layout of power energy, resulting in big social effects. The emphasis of this paper is to discuss the impacts in management.

\section{Safety and efficiency of automated driving}

\subsection{Traffic safety}

According to NHTSA's investigation: nearly $90 \%$ accidents resulted from driver's mistakes [4] including distraction, speeding, drunk driving and situation misjudging. Compared with manned driving, unmanned driving has obvious advantages:

- Accurate operations

Self-driving car can strictly implement orders, which determines that it can avoid human errors and reduce the possibility of accidents. According to IIHS's estimation [5], accident rate is possible to be reduced by $1 / 3$ if all cars are equipped with lane departure warning, overspeed warning, distance warning and adaptive headlamps. - No physical defects

Self-driving car is sensitive and fatigue will not appear, which means great reduction of accidents.

Of course, it should be recognized that potential security risks also exist if autonomous car is applied, such as sensor errors or software mistakes. However, the probability of machine failures is smaller than that of human mistakes, and it is easier to avoid machine failures. In summary, man-made traffic accidents will be greatly eliminated and traffic safety will be improved.

\subsection{Traffic efficiency}


In addition to excellent safety performance, traffic efficiency is also of great advantages [6].

\subsubsection{Fully self-driving traffic}

The advantages of increasing traffic capacity and shortening commuting time are obvious when road is universally occupied by self-driving cars. Firstly, the sensitivity and accuracy of computer system are far beyond that of human. Secondly, utilizing connected car, information can be transferred among vehicles.

\subsubsection{Mixed traffic}

This will presumably be the normal state, in which conventional and automated vehicles interact with each other, for quite some time even. It is, therefore, of great practical significance to gain a precise understanding of traffic efficiency under this situation.

Wagner. P [7] constructed a mixed traffic flow model, and analyzed the impacts of the introduction of driverless cars on traffic by simulation experiments. The study showed that under the existing traffic conditions, the greater the proportion of autonomous cars, the more significant increase in capacity and the higher passing rate appear. But compared to the pure self-driving environment, mixed traffic still has the following problems:

- Self-driving cars cannot effectively reduce the startup delay at a crossing. This is because the traditional car delay breaks the continuity of autonomous vehicles.

- In the mixed traffic, traditional cars' limitation in carfollowing determines there will be no high-speed highdensity traffic queue (which can be also called platoon) which can only be realized in a pure autonomous environment.

Therefore, in the mixed traffic, its greater advantage is excellent safety, and the followed is efficiency.

\section{Traffic management}

While self-driving vehicles have shown great advantages in terms of safety and efficiency, the realization of these advantages requires not only advanced technology, but also corresponding traffic management approaches. However, the safety and efficiency of self-driving vehicles are limited by the existing traffic facilities, laws and regulations nowadays. In the future, along with the promotion of autonomous vehicles, the forms, the using rules of transport infrastructures and the accident liability must be changed accordingly.

\subsection{Infrastructure management in fully autonomous traffic}

\subsubsection{Intersection management}

As a major bottleneck for traffic efficiency and safety, intersection attracts many researchers' attentions all the time. In most existing intersections, traffic lights are installed to coordinate conflicting traffic flows. However, the efficiency and safety of such system is doubted: $44 \%$ of collisions in the U.S. are within the intersection area and delays induced by traffic lights can be high [8].

In 2008, Dresner and Stone [9] of Texas University at Austin proposed the concept of intelligent intersection. Automated vehicles are coordinated to cross intersections without traffic lights, fully utilizing the advanced sensing, communication and manoeuvre capacities of automated vehicles. This concept is widely accepted by researchers for its efficiency improvements (throughput, average delay, etc.)and many improved approaches are proposed [10-17], which can be categorized into planning-based approaches [9-12] and hybrid approaches [13-17].

1) Planning-based approaches

In planning-based approaches, an intersection controller finds collision-free trajectories for all vehicles. Then vehicles should follow the trajectories to cross the intersection. Any deviation from planned trajectories may cause collisions. The difficulty of planning method is to generate trajectories for all cars, which is a great challenge to the computing capability of intelligent intersection. J. Lee et al. [12] argued trajectory generation is a complex large-scale nonlinear constrained optimization problem that can be performed using optimization methods, such as Active-set Method [18], Interior Point Method [18] and Genetic Algorithm [19]. Dresner and Stone [9] modeled a "reservation based" approach to intersection management enabled by driverless technology. The vehicles could reserve trajectories from the intersection controller and the controller will centrally decide to accept or deny the reservations. These centralized, planning-based approaches are quite efficient, while autonomous cars' behavior is not subject to their own control and autonomy cannot be ensured. However, the unique needs of each vehicle are difficult to integrate into the planning method as this will necessarily introduce a large number of parameters, adding more complexity to already complex system. So the vehicle can only be fully subject to the intersection controller. The egoistic needs of each individual vehicle might be ignored.

2) Hybrid approaches

- Priority-based approaches

Hybrid approaches plan some high level priority relations defining the relative orders of vehicles. Obeying these relations, vehicles are able to cross intersections safely. L. Makarem et al.[13] proposed an approach based on navigation function to define priority. When the two cars are both in the crossroad area, the vehicle's navigation function will send their own route information to the intersection controller, and the probability of collision between the two cars will be calculated according to the vehicles' locations, driving directions and speed. If there is a possibility of collision, the vehicle nearer the collision point will be assigned greater priority and be informed to accelerate while the other one will slow down to avoid crash. In figure 1. there is a collision point between vehicle one and vehicle two. Consequently, vehicle two should be accelerated and vehicle one should slow down. If priority relations are properly defined in 
such approaches, the balance can be achieved between cooperation and egoistic need.

The method may avoid collision under normal situation, except for some extreme cases. For example: vehicles collide as unexpectedly braking due to the presence of a pedestrian. To solve such problems, $\mathrm{H}$. Kowshik et al.[14-16] adopted a more control theoretical approach to formulate safety constraints. A bad set that comprises all collision configurations can be defined. Vehicles are then controlled in a way that they will always avoid the bad set.

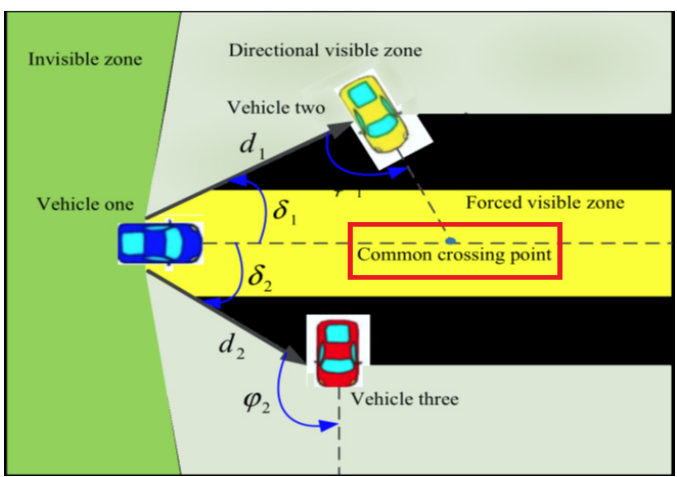

Figure 1. Intersection control units calculating the probability of collision [13]

- Autonomous intersection models

Arnaud et al.[8] established a autonomous intersection model shown in Figure 2. which includes:

- A roadside unit as the intersection controller is responsible for generating the permissions for the vehicles entering the intersection;

- Inner dotted box represents the intersection area where vehicles without permission will not be admitted;

- Outer area refers to the cooperative area, where vehicles start to communicate and cooperate with the intersection controller.

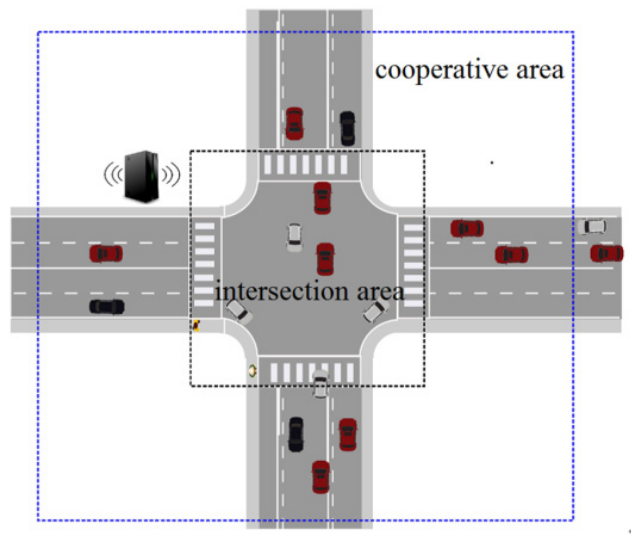

Figure 2. Schematic diagram of the autonomous intersection

Vehicles that enter the cooperative area notify the intersection controller with their presence and the controller adds it to the waiting sequence. At the same time the controller maintains a permissions list. The controller works in discrete time. At each time step, the intersection controller will add several vehicles to the permissions list from the waiting sequence, then the vehicles are permitted to pass through the intersection. Once passing through the intersection, they will be released from the permission sequence. In addition, by adjusting the speed, the vehicles can cross the intersection area without waiting, improving passing efficiency.

Three organizations, including MIT, have jointly created Slot-Based Systems [20], which are similar to those commonly used in aerial traffic: (1) Based on welldesigned scheduling algorithm, the systems are able to allocate safe arrival time slots at cross areas to vehicles;(2) Vehicles control their speed to reach the intersection at the beginning of the assigned time slot, avoiding traffic conflicts and without stopping to wait. Both Slot-Based Systems and the above autonomous intersection model proposed by Arnaud focus on the interaction between vehicle and intersection controller. Though the two models share similarity in control methods, there are still differences:

- In both models, vehicles experience the same two states: the first is waiting and controlling the speed, the second is through the intersection. In the autonomous intersection model established by Arnaud, the above two states are clearly distinguished by the intersection controller for the clear division of the cooperative area and intersection area, while the boundary between these two states is not obvious in Slot-Based Systems.

- The order of permission assignment between these two models is quite different. The autonomous intersection model is controlled by the intersection controller. Thus the vehicle first controls the speed to wait for permission. After admitted, the vehicle can finally cross the intersection; In Slot-Based Systems, the vehicle first obtain the access, and then adjust speed to cross the intersection.

The above analysis shows that traffic flow in SlotBased Systems is more smooth. Forming platoons of vehicles and serving all vehicles in the platoon before giving way to a conflicting flow is more efficient from a capacity point of view. Based on the generalized queue theory, the researchers found that the Slot-Based Systems capacity can be doubled and the delay can be significantly reduced compared to the traffic-light controls [20]. But the premise of Slot-Based Systems is that the roads are basically occupied by autonomous cars, thus there may be decades to achieve this system.

\subsubsection{Parking management}

The emergence of driverless vehicles will make urban parking management model adjust accordingly. The ideal management model is: when owner arrives at the destination, the autonomous car can find their own safe and reliable parking space without human operation.

Volkswagen has already taken some projects testing parking in garages with autonomous vehicles [21], [22]. The driver hands the vehicle over at the garage entrance. The parking function is activated with a smartphone app. The car receives the route data to the nearest available space from a central garage computer via WLAN and drives to it autonomously. 
The possibility of saving space is named as an essential argument for the use of automated parking systems. These should be made more space-efficient, primarily by replacing ramps and aisles with lift shafts, and lowering storey heights, but also by increasing parking density [23], [24]. Kowalewski.S [25] reckoned on up to $60 \%$ more parking spaces on the same area by using automated parking. It will relieve parking pressure in some functional centers (urban commercial centers, scenic areas, etc.) and greatly reduce the cost of urban construction and parking facilities [25-27].

\subsubsection{Pedestrian crossing management}

Autonomous driving leads to expectations that capacity on transport routes will be freed up with platoon. Fernandez [28] assumed vehicle density increase up to $500 \%$. Brownell [29] estimated over $250 \%$ for highways and some $180 \%$ for inner-city streets.

High density means that the area required for flowing traffic could be reduced, by reducing the number of lanes [30]. Lane width could also be cut in comparison to the present size, due to the accurate lane-keeping technology of autonomous vehicles. These could have an impact on transport users such as pedestrians. Although the distance of crossing street decreases, densely flowing traffic may make it tougher for pedestrians. In order to guarantee the conveniences of pedestrians, the installation of intersection-free crossings such as under and overpasses would be a necessary consequence.

\subsubsection{Other transportation facilities management}

Automated vehicles propose specific demands for road quality, traffic conditions, traffic signs, safety facilities, etc. [31]:

- Lane markings need to be clearly identifiable by automated driving systems and their vision sensors. For lane assistance systems, the quality of lane markings is crucial. Lane markings should be detected by the employed sensors irrespective of weather conditions (e.g. rain,snow, etc.) or road conditions (e.g. presence of cracks, potholes).

- The color, luminance and shape of the various marks must be readable for vehicle sensors

- Old lane marking remnants should be well removed, so they do not create any confusion.

- Defined and visible road profile and continuous road edges or kerbs are needed

- Electronic speed limitation is indispensable.

- Roadside V2I/I2V communication infrastructure (via wireless G5 or cellular networks) should be employed.

These requirements are found in the self-driving vehicle tests. The solutions to these problems can also accelerate the pace of putting automated vehicles into operation.

\subsection{Infrastructure management in mixed traffic}

At present, most of the researches on self-driving traffic management are concentrated in fully self-driving traffic, and there are few studies in mixed traffic. The existing strategy is to separate unmanned vehicles from manned vehicles, thus effectively taking the advantages of autonomous vehicles. One way to achieve this aim is to create an autonomous vehicle dedicated lane.

As the efficiency of the traffic depends largely upon the on-ramps and off-ramps of freeway and the signalized intersections on the urban roads, the focus of the mixed traffic management research is on the two issues. Bernhard [32] has conducted some studies on them.

\subsubsection{Motorways}

The on-ramps and off-ramps are the most important elements that affect the traffic efficiency of motorways, and the following management measures can be carried out [32]:

- One measure is looking forward to further developing already-emerging technical solutions with assistance functions, such as the merging assistant, particularly in regard to the possibilities of machine cooperation.

- Solutions for adjustments of facility structure and regulatory mode are still to be developed. For example, one interesting scenario is where autonomous traffic can be directed onto separate lanes between motorway intersections. This separation is then removed in intersection areas where autonomous and humancontrolled vehicles drive in all lanes and each may perform all maneuvers (autonomous, highly assisted or human-driven) at a predetermined low speed. It will be ensured that autonomous vehicles can form platoon, without affecting the vehicle to change route.

\subsubsection{Urban roads}

Urban traffic flow is complex, so the rules of right of way should still be adhered to under mixed traffic. Signal control system could set a separate phase for autonomous vehicles in which self-driving vehicles from all directions can cross the intersection simultaneously [32]-the maneuvers of the conflicting flows in the intersection area would be negotiated independently by the autonomous vehicles. All other road users would be controlled by the existing signal phases. By such differentiated management strategy, it is expected to maximize the automated driving advantage under mixed traffic.

\subsection{Laws and regulations}

A crash involving Tesla Model S occurred on May 7, 2016, Florida, USA, due to the autopilot's failure of identifying the front vehicle, which resulted in Tesla owner's death. NHTSA is still conducting technical verification on the same type of Tesla Model S, in order to affirm who is responsible for the accident. As the first fatal traffic accident involving automated car in the U.S, not only reveals that the driverless technology is not completed, this incident also indicates that before the application of self-driving car, the principle of driverless 
traffic accident liability division and other related regulations are necessary to be formulated in advance.

\subsubsection{Accident liability division}

Since both car manufacturers and car users are involved in the driverless traffic accident, the division of responsibility should be respectively discussed .

1) Responsibility of car manufacturers

Under the conventional traffic, once an accident happened, it is usually the driver not the car manufacturer who will be held accountable because car is completely controlled by the driver. On the contrary, after the application of driverless cars, they can control themselves and the drivers no longer have the access. The accident involving self-driving car is usually caused by the defects of the car itself, so it is unreasonable that the driver should be completely responsible for the accident. At that time, the car manufacturer will take more product liability for the accident compared to the previous situation [4].

The product liability of car manufacturers can be judged by two principles, including strict product liability and negligence product liability.

- Strict product liability: As long as the existence of defects leads damage to men and property, The car manufacturer will assume the reparation liability wherever the fault is subjective or not, except for the statutory exemption mistakes. Under the principle, the consumer will get compensation as long as he proves the relationship between the product and the manufacturer and points out the defects of the product, which is beneficial for the consumer to cite evidence.

- Negligence product liability: This principle, bringing great difficulties to the consumers, requires the car users not only to point out the flaws, but also to prove that the producers do make a subjective mistake, which is demanding for the consumers to cite evidence.

If autonomous car is applied, the principle of strict product liability can meet the psychological needs of consumers [33]. In the manufacturers' point of view, the cost of taking liability, brought by this principle, may weaken the enthusiasm of driverless car manufacturers. In fact, manufacturers, including Volvo, Google and Mercedes, are committed to compensate for the loss of driverless car accidents. Taking the responsibility initiatively will eliminate consumers' doubts about the new technology and help to promote the automated vehicles. In addition, the increased cost can be transferred to the price of the car.

2) Responsibility of car users

The tort liability of car users can be also judged by two principles: no fault liability and fault liability.

If the principle of fault liability is insisted on, the injured party will have to prove that the driverless car user had made a subjective fault. As the user was not at fault, the victim can do nothing but turn to sue the manufacturer for product liability. Under that circumstance, they have to confront expensive litigations, lengthy trial period and adduction difficulty. In contrast, non-fault criterion liability is easier to implement as victims can receive compensation directly from insurance companies and the litigation expense is eliminated. In conclusion, the principle of no-fault liability is more applicable to the tort liability authentication of driverless vehicle users.

\subsubsection{Traffic laws}

In fact, there have been some tentative laws for autonomous vehicles. In 2011, Nevada took the lead in passing legislation, allowing self-driving vehicle tests to be carried out. Subsequently, Florida, California, Washington, the District of Columbia etc, have also allowed the tests in their states. Nevada has also proposed rules for driverless vehicle sellers and users to ensure the reliability of vehicles [2]. Sellers must obtain certificates of compliance from car manufacturers or related technology accreditation bodies and users need to obtain specific licenses. However, these regulations do not illustrate the principle of liability division for the accident clearly.

On December 9, 2016, the first comprehensive statute in the United States, covering the test, use and sale of driverless vehicle, was promulgated in Michigan. The law stipulates that the liability in an autonomous car accident will be deduced based on the principle of nofault in the Michigan Insurance Act, and the loss will be paid by the insurance companies. At the same time, the manufacturers will be held accountable under the product-liability law. The establishment of the law, setting a benchmark for the legislation of administration over autonomous vehicles, stimulates the initiation of lawmaking in other regions and benefits the further development of driverless technology.

\section{Conclusions and prospect}

This paper reviewed the development of self-driving technology and discussed the advantages of driverless vehicles in safety and efficiency. On the basis of the above discussion, it is recognized that appropriate traffic management measures are still needed to guarantee the superiorities. Therefore, this article, mainly from traffic facilities and laws, summed up the following key points on the autonomous traffic management.

1) Driverless vehicles can not only effectively improve traffic safety, but also significantly enhance traffic efficiency. However, it is worth noting that, in the mixed traffic flow combining conventional, connected, and automated vehicles, the advantage of traffic efficiency is not so distinct that setting dedicated lanes are necessary to maximize the preponderance of self-driving vehicles.

2) In the case of coexistence of autonomous vehicles and manned vehicles, the researches focused on the onramps and off-ramps of freeways and the signalized intersections on urban roads. For ramps of expressways, merging assistant as well as adjustments of facility structure and regulatory mode will take effect. For urban signalized intersections, exclusive signal phase for driverless vehicles can take full advantage of them and enhance traffic efficiency adequately. 
3) If self-driving vehicle is applied universally, the management pattern of intersection will be changed completely, from traffic signal into intelligent intersection. The control of intelligent intersection can be divided into planning-based approaches and hybrid approaches.

- Planning-based approaches: The approaches manage intersections by planning collision-free trajectory.

- Hybrid approaches: This kind of approaches includes priority-based approaches and autonomous intersection models. The priority-based approaches define relative orders and bad-sets to control vehicles, while the autonomous intersection models assign permissions to those vehicles through intersections.

Automated intersections adapted to autonomous technology not only strengthen safety, but also reduce delays and the pressure of urban traffic capacity. Among above approaches, autonomous intersection models may be optimal for the reason that autonomous intersection models, different from planing-based approcahes, take the egoistic needs of each individual vehicle into consideration and deceleration is not required in the model compared with priority-based approaches. Moreover, in the autonomous intersection models, the vehicle will running more smoothly in Slot-Based Systems without obvious boundary.

4) With the development of self-driving technology, other transportation facilities, including parking lots, pedestrian crossing facilities, signs, markings, and communication facilities, also need to adapt themselves to the automation demands. For example, intelligent parking lots are required to achieve automatic parking management.

5) In terms of laws and regulations, car manufacturers will assume more product liability than they do now. What's more, principle of no-fault liability is more suitable for both manufacturers and users.

Researches on driverless traffic management remain to be further studied, although plenty of corresponding traffic management approaches have been proposed. Moreover, there are many other obstacles to the promotion and implementation of autonomous vehicles, including the immaturity of technology, the resistance of the traditional automotive industry, and ethical difficulties. Once these problems are resolved, it will not be far for self-driving vehicles to enter the market.

\section{References}

1. D. C. National Highway Traffic Safety Administration, U. S. Department of Transportation (2013).

2. Adeel Lari, Frank Douma \& Ify Onyiah.16 Minn. J. L. Sci. \& Tech. 735 (2015)

3. Fung. Technical Report (2013).

4. NHTSA.Preliminary Statement of Policy Concerning Automated Vehicles (2013)

5. M. James Anderson, Nidhi Kalra, D. Karlyn Stanley, Paul Sorensen, Constantine Samaras, Oluwatobi A.
Oluwatola. Autonomous Vehicle Technology. A Guide for Policymakers (2014).

6. Todd Litman,TRB, 15-3326 (2016).

7. Wagner, P. Traffic control and traffic management in a transportation system with autonomous vehicles, In Autonomous Driving (2016).

8. Arnaud De La Fortelle, Xiangjun Qian. ITS World Congress (2015).

9. K. M. Dresner, P. Stone, J. Artif. Intell. Res.(JAIR), vol.31, pp. 591-656 (2008).

10. K. Dresner, P. Stone, Springer, pp. 129-138 (2006).

11. I. H. Zohdy, R. K. Kamalanathsharma, H. Rakha, ITSC, pp. 1109-1114 (2012).

12. J. Lee, B. Park, K. Malakorn, J. So, Transp. Res. Part C Emerg. Technol, vol. 32, pp. 193-206 (2013).

13. L. Makarem, M. H. Pham, A. G. Dumont, D. Gillet, TRB (2012).

14. H. Kowshik, D. Caveney, P. R. Kumar, Veh. Technol. IEEE Trans, vol. 60, pp. 804-818 (2011).

15. K. D. Kim, P. R. Kumar, IEEE Trans. Automat. Contr., vol. 59, pp. 3341-3356 (2014).

16. M. R. Hafner, D. Cunningham, L. Caminiti, D. Del Vecchio, IEEE Trans. Intell. Transp. Syst., vol. 14, pp. 1162-1175 (2013)

17. M. A. S. Kamal, J. Imura, A. Ohata, T. Hayakawa, K. Aihara, ITSC, pp. 922-927 (2013).

18. J. Nocedal, S. Wright. Numerical Optimization. Springer (2000).

19. K.Ozbay, H. Yang, B. Bartin, S. Mudigonda. Transportation Research Record, pp.105-113 (2008).

20. Tachet R, Santi P, Sobolevsky S, Reyes-Castro LI, Frazzoli E, Helbing D,et al. PLoS ONE (2016)

21. Continental AG: Der Fahrer entscheidet, das Auto übernimmt (2014).

22. Audi: Automatisch ins Parkhaus (2012).

23. Fagnant, D. J. Kockelmann, Eno Foundation (2013).

24. Irmscher,I. (n.D.): Benutzerfreundliche automatische Parksysteme - Besondere Anforderungen -Planung Einsatz.

25. Kowalewski, S. Überlassen Sie das Parken Ray. Deutschlandradio Kultur (2014).

26. W. J. Mitchell, E. Boronni-Bird, L. D. Burns, The MIT Press (2010).

27. LEG Stadtentwicklung GMBH: Mobilitätshandbuch Zukunftsstandort Phoenix West.Dortmund (2008).

28. C. K. Brownell: Shared Autonomous Taxi Networks: An Analysis of Transportation Demand in NJ and a 21st Century Solution for Congestion (2013).

29. C. K. Brownell \& A. Kornhauser, TRB (2013).

30. P. Fernandes, IEEE Transactions on Intelligent Transportation Systems, vol.13 (2012)

31. Isabela Mocanu, Philippe Nitsche, Peter Saleh. 25th PIARC World Congress (2015).

32. B. Friedrich, "The Effect of Autonomous Vehicles on Traffic" In Autonomous Driving, Chapter 16, pp. 317-334(Springer Berlin Heidelberg, 2016).

33. S. Stephen. "Product Liability Issues in the U.S. and Associated Risk Management" In Autonomous Driving, pp.523-570 (2016). 